\title{
A practitioners approach to individual claims models for bodily injury claims in german non-life insurance
}

\author{
Marcel Wiedemann (iD $\cdot$ Daniel John
}

Accepted: 15 December 2021 / Published online: 10 January 2022

(C) The Author(s) 2022

\begin{abstract}
The aim of our paper is to discuss the difficulties non-life actuaries are currently facing from a practical point of view. Based on this, we show that individual claims models are the key to address these difficulties and discuss how such models give actuaries a new and very powerful tool to explore further fields of application. Moreover, we address a very essential question: What data is needed for developing individual claims models? For bodily injury claims in German motor liability insurance, we shall derive specific attributes based on a detailed discussion of the legal background. All our ideas are based on practical experience for a large German motor insurance portfolio.
\end{abstract}

\section{Die Entwicklung von Einzelschadenmodellen für Personenschäden in der Schaden-/Unfallversicherung aus praktischer Sicht}

Zusammenfassung Im unserem Artikel diskutieren wir die praktischen Probleme, die Schaden-/Unfall-Aktuare gegenwärtig bewältigen müssen. Wir zeigen, dass Einzelschadenmodelle der Schlüssel zur Lösung sein können und diskutieren, wie Aktuare sich mittels solcher Modelle viele neue und spannende Einsatzgebiete erschließen können. Wir beschreiben ebenfalls, welche konkreten Daten für die Entwicklung von Einzelschadenmodellen nötig sind. Für Personenschäden beleuchten wir die rechtlichen Hintergründe und leiten daraus die benötigten Variablen und Attribute zur Entwicklung von Einzelschadenmodellen ab. Unsere Ausführungen basieren auf praktischen Erfahrungen für ein großes deutsches Kraftfahrt-Portfolio.

\footnotetext{
Marcel Wiedemann $(\bowtie)$

Hochschule Esslingen, University of Applied Sciences, Kanalstraße 33, 73728 Esslingen am Neckar, Germany

E-Mail: Marcel.Wiedemann@hs-esslingen.de

Daniel John

HUK-COBURG Versicherungsgruppe, Bahnhofsplatz, 96450 Coburg, Germany

E-Mail: Daniel.John@huk-coburg.de
} 


\section{Introduction \& background}

In recent years non-life reserving actuaries had to face several major challenges. Due to Solvency II, calculations need to be more detailed, of higher quality, and completed in a significantly shorter amount of time. Moreover, actuaries are required to have a better understanding of the data used and their origin (that is, the processes generating them). At the same time, increased competition in the insurance market led to the need for a better understanding of the economic situation of insurance companies, entailing the necessity for a better understanding of claims provisions and their development over time (for they are usually the most important liability and, hence, have a large impact on the economic situation).

In this environment the role of non-life reserving actuaries has become much more challenging. They now need:

- A clear vision of how to connect all different processes as well as how to actively form and shape their insurance company using data-based methods. Especially designing innovative steering concepts supporting management as well as operational levels is of utmost importance.

- Communication skills, the ability to bridge the gap between the operational level (where the input for actuarial reserving comes from) and the management level (where actuarial results are used for steering purposes) as well as the will to actively form, shape, and support management decisions have become essential skills in recent years.

- A sound understanding of methods and models they can use to accomplish their mission.

This new situation forced actuaries to rethink their processes, workflows, tools, and techniques, in order to be able to meet the described challenges and fulfil their new role. Reserving, pricing, risk management, internal modelling, controlling, forecasting, claims steering, etc. can no longer be thought of as different processes but have become closely connected and, hence, need a more comprehensive approach and a common basis. However, developing such a comprehensive approach to all actuarial processes usually proves quite difficult in practice, because by using standard actuarial methods based on aggregated triangle data (such as chain-ladder method on paid/incurred claims triangles) it is very difficult to obtain results which are detailed enough to be utilized in all following processes consistently (for background information on reserving methods on aggregated triangle data see Mack 2002).

As a solution to the aforementioned challenges, so-called individual claims models (which aim to model best estimate provisions for individual claims as opposed to modelling the entire portfolio) have been discussed increasingly widely amongst actuaries in recent years (see for instance Antonio and Plat 2014; Pigeon et al. 2013; Wüthrich 2018). These discussions, however, usually focus entirely on mathematical methods and techniques and there appears to be an aspect that is sometimes overlooked but very essential: What exact claims data is needed for "good" individual claims models? It seems obvious, that the best mathematical methods and techniques will be powerless without "adequate" claims data to work on. But what do we mean by "good" models and "adequate" data? These are more than just technical aspects 
and will strongly depend on what one wants to achieve using individual claims models. To support claims steering for instance, they will have to be a lot more detailed than just for reserving or pricing. This shows that developing individual claims models requires a much wider and a more comprehensive approach (going far beyond mathematical methods and techniques). To this end, a clear vision of how these models should fit into an insurance company and its steering hierarchy is absolutely essential.

The focus of our paper is to motivate individual claims models and to give an answer to the very essential question of what data is needed for modelling in case of bodily injury claims in German motor liability insurance. Since provisions for bodily injury claims usually constitute the largest share of claims provisions in motor insurance, modelling and understanding them is of utmost importance for German non-life actuaries. All our ideas are based on practical experience for a large German motor insurance portfolio. In the last years we have developed individual claims models for many lines of business, and they are currently being deployed to support actuarial reserving as well as claims handling.

The structure of our paper is as follows. In Sect. 2 we shall demonstrate the practical difficulties one encounters when working with claims triangles and standard reserving methods such as the chain-ladder method. Based on a concrete example we shall discuss the challenges of predicting a portfolio reserve based on aggregated data and show that it is very hard to actually use these results in relevant areas such as risk management, claims steering or claims handling. This will show the need for a more comprehensive approach to all actuarially related processes, and it will become clear that individual claims models might be the desired solution.

In Sect. 3 we focus on deriving the exact data needed for individual claims modelling in the case of bodily injury claims. We start by giving a detailed overview of claims handling as well as the legal background for bodily injury claims in German motor liability insurance. Based on this, we can derive necessary attributes needed for modelling. We believe that this approach is valid in general and should always be the first step in the development of individual claims models. In Sect. 4 we discuss the consequences for non-life actuaries and focus on open questions and possible areas for further research. Our paper finishes with a conclusion in Sect. 5.

\section{Close-up: the challenges of non-life actuaries}

We have just described that actuaries are currently facing quite a number of changes to their job description. In this part we aim to discuss a concrete example, showing exactly the kind of problems they need to deal with.

Currently, actuaries perform their calculations mostly using traditional methods (such as chain-ladder method) based on aggregated triangle data (paid or incurred). This setup makes it very hard to bridge the gap between the aggregated view (used for top-level management purposes) and the operational view (used for operational risk management, claims steering, etc.). Deriving detailed information-relating directly to operational business transactions - from aggregated methods is almost impossible, because by aggregating all details are lost and cannot be retrieved. 
Let us assume we are trying to calculate the best estimate reserve for a (German) motor liability portfolio based on aggregated data. As a first step, one would usually split the data into two parts: non-bodily injury claims and bodily injury claims. Based on this, the second step would then be to calculate the best estimate reserve for both sub-portfolios individually. In our example, we want to focus on bodily injury claims only, since they usually form the largest share of all liabilities. Moreover, let us assume for simplicity, that bodily injury claims consist of just two compensation components: damages for personal suffering and loss of earnings. In practice, there are obviously more components (for further details, see Küppersbusch and Höher (2016) or Sect. 3.2 for a summary). However, these two components will already be enough to demonstrate the main problems with aggregated methods (things will only become more involved when adding further compensation components). Let us first take a closer look at both components: Damages for personal suffering aim to compensate for the suffering endured during or in the aftermath of an accident. It will hence be strongly dependent on the severity of an accident and the injuries resulting from it. Personal suffering will usually be a short tail position, settled within a few years after the accident (normally once the injuries und their severities are known). Damages for loss of earnings aim to compensate for the lost earnings (past and future) caused by an accident (which usually forces the claimant to interrupt their employment, at least temporarily). It will hence be strongly dependent on the severity of injuries but also on the occupation as well as wage and age of the claimant (for further details, see Küppersbusch and Höher (2016) or Sect. 3.2 for a summary). Loss of earnings will usually be a long tail position, since the compensation might run until retirement age of the claimant (although lump sum settlements are possible).

One can already see, that claims triangles composed of claims consisting of the aforementioned components must be distorted in some way, since they are very different and depend on different factors. This will especially be the case, if claims (or rather the internal and external effects influencing them) change considerably over time (for instance due to legal changes ${ }^{1}$ ). The general assumption in practice that on average things will not change is usually not valid (for mathematical background on aggregated triangle methods see Mack 2002). As a consequence, the applicability of standard reserving methods on aggregated data seems restricted. At the same time, it seems clear that an understanding of the characteristics of claims and its components on a detailed level (as described above), makes it possible to develop more suitable models (on the level of individual claims) which might replace standard aggregated methods.

One might of course argue that a further splitting-up of claims data would yield more homogenous groups and, hence, solve the problems just described. In practice, however, there are two main objections to this: Firstly, detailed data is usually not available. Normally, paid and incurred data will only be available in total (and not split up in all its compensation components). This means, of course, that the segmentation into damages for personal suffering and loss of earnings as described

\footnotetext{
${ }^{1}$ Examples might be the introduction of Hinterbliebenengeld in 2017 (as part of damages for pain and suffering), changes of retirement age or changes in health insurance coverage (for example the introduction of DRG's in 2003/2004).
} 
Table 1 Injury types and cashflows per component

\begin{tabular}{lllllll}
\hline Type & Component & 1st year & 2nd year & 3rd year & 4th year & 5th year \\
\hline I: Slight & Personal suffering & 10,000 & 0 & 0 & 0 & $\ldots$ \\
Injury & Loss of earnings & AW & $0.2 \bullet$ AW & $0.2 \bullet$ AW & $0.2 \bullet$ AW & $\ldots$ \\
II: Medium & Personal suffering & 0 & 50,000 & 0 & 0 & $\ldots$ \\
Injury & Loss of earnings & AW & AW & $0.5 \bullet$ AW & $0.5 \bullet$ AW & $\ldots$ \\
III: Heavy & Personal suffering & 0 & 0 & 150,000 & 0 & $\ldots$ \\
Injury & Loss of earnings & AW & AW & AW & $0.9 \bullet$ AW & $\ldots$ \\
\hline
\end{tabular}

above is completely artificial. Secondly, and even more importantly, if one could split up claims data further, one would end up with the same problem (of having to split up even further). Say, for instance, we consider a triangle just consisting of loss of earnings compensations. In this case, claims of claimants with age 25 earning $2000 €$ might develop very differently to those of claimants with age 55 earning $5000 €$ (reasons being different recovery of health, life and job situation, etc.). So, one would need to split up even further and so on, which will eventually lead to just analysing individual claims. This already marks an important point:

Separating claims portfolios into homogenous groups will almost inadvertently converge towards (or get close to) individual claims.

Let us consider the following example to demonstrate the points just made. For simplicity we assume that there are three types of bodily injuries (classified by severity/consequences of injury, in practice this might be given by the reduction in earning capacity, see Sects. 3.2.1 and 3.2.3) with the following characteristics and annual cashflows (where AW denotes the annual wage of the claimant, payments being made annually in arrears, cashflows starting at time of incident not reporting year) (see Table 1).

Just three types of claims without variation in compensation and development (for each type) are obviously a significant simplification, but enough for demonstration purposes. In practice, things will obviously be a lot more difficult (see Küppersbusch and Höher (2016) or Sect. 3.2 for a summary).

The assumptions above are derived from practical experience and cover the main characteristics of both components. The ideas behind them are as follows: The heavier the injury and its consequences, the longer it takes to finally assess its impact. Hence, compensation for personal suffering will be delayed. Moreover, incapacity for work will take longer and restrictions to work (later on) will be greater.

In practice, additional factors will come into play, making the situation a lot more difficult. Some of these factors are:

- Claims handling processes might change considerably over time delaying or accelerating payments (hence affecting cashflows).

- Legal changes, claimants' behaviour, seasonal effects, etc. will affect claims amounts as well as cashflows.

- Claims will obviously be reported at different times during the year (the table above defines what happens after reporting) and when aggregating them into 
claims triangles this will play a crucial role (by adding an additional shift to cashflows).

- Claims might change their types (I-III) during development for instance due to better or worst healing processes etc.

- Due to inflation, personal suffering payments will increase over time.

- Due to medical progress, damages for loss of earnings might change considerably over time (since physical conditions might be restored faster and to a higher degree).

- Loss of earnings will not only depend on the severity of an accident, but also on factors like age and occupation of claimants (see Küppersbusch and Höher (2016) or Sect. 3.2 for a summary). For instance, labourers who depend on their physical capabilities (more than employees for instance) will obviously be more affected by an accident. Moreover, younger claimants have a tendency-depending on the injuries - to recover better and faster.

- Lump sum settlements of loss of earnings claims will have a huge impact on cashflows (time of settlement, interest rates, etc.).

Now, a claims triangle aggregated from these claim types will mainly depend on the shares of different types per year (which might be highly volatile). For simplicity we shall assume that all claims happen on January 1st (which is of course a crude simplification but suffices for demonstration purposes).

Let us assume, the shares have developed over the years in the following manner (see Fig. 1).

Assuming constant shares of claim types over time is definitely not realistic, in practice there will be (occasionally huge) variation.

This yields the following chain-ladder development factors for damages for personal suffering and loss of earnings (see Figs. 2 and 3).

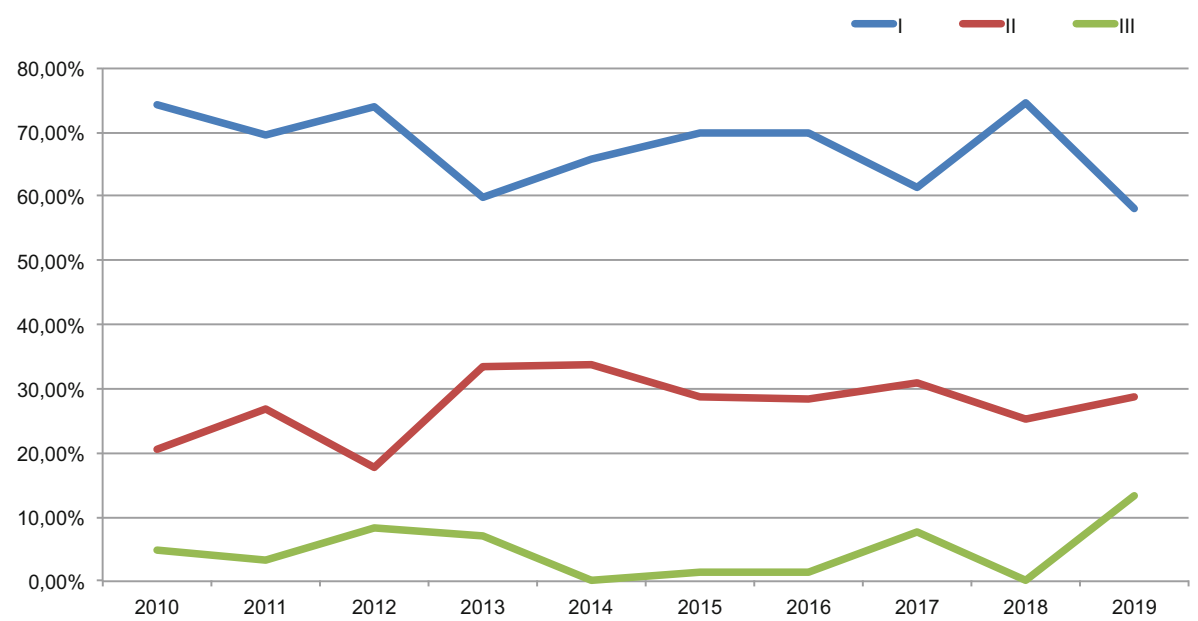

Fig. 1 Shares of claim types 


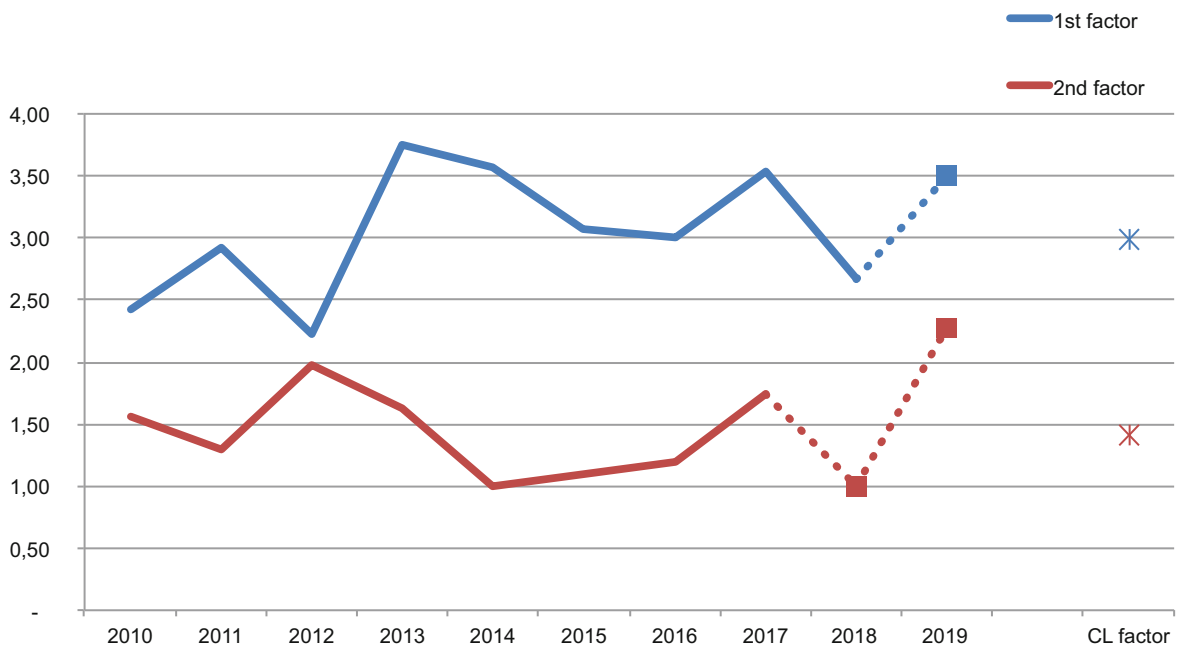

Fig. 2 Development factors personal suffering

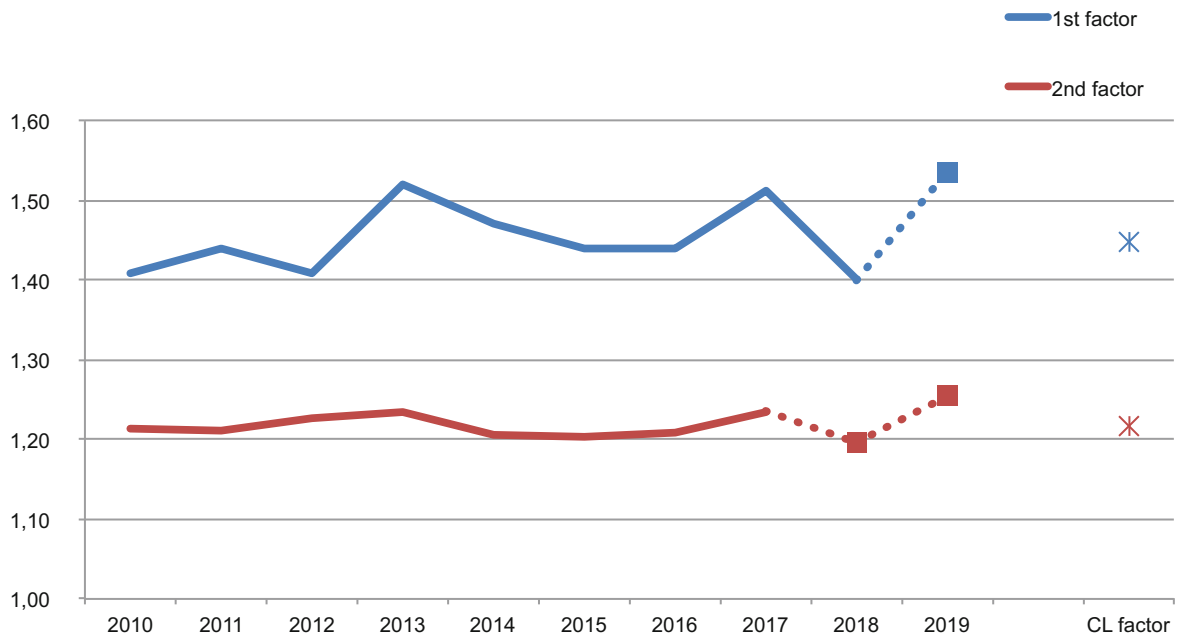

Fig. 3 Development factors loss of earnings

Aggregating both compensation components, we obtain the following chain-ladder development factors (see Fig. 4).

In this small example, all that varied over time were the shares of different claim types (which is, of course, a simplified assumption), making it already very hard to interpret the development factors (and their volatility) without knowing what is really going on in the background on the level of individual claims (in practice, as discussed above, many more factors will vary over time, making things even more difficult). We see that the chain-ladder predictor is (most of the time) far from the "real" development factor (even if it is close, this will be by accident). The reason is simple: Years in the past are not necessarily relevant for future behaviour, since 


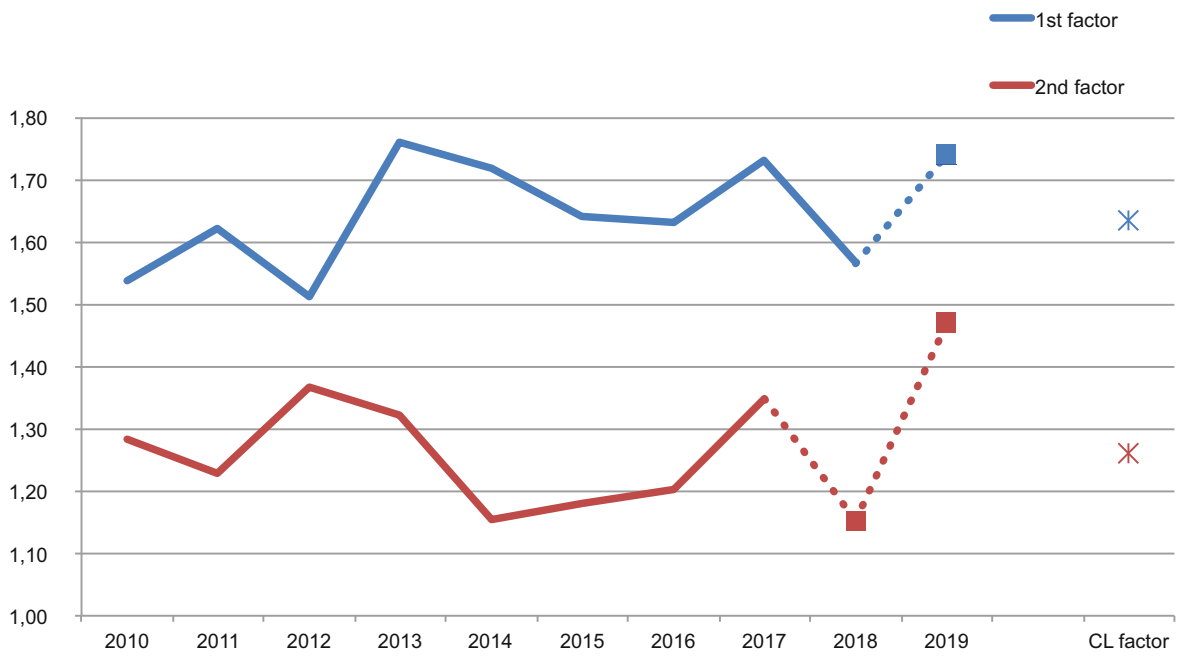

Fig. 4 Development factors portfolio

the mixture of claim types (and their characteristics) may have changed and, hence, averaging over the past is just not appropriate. This is the main reason why it is hard to estimate reserves just by using aggregated data of the past.

As mentioned before, further segmentation of claims triangles will in practice not yield better results in general (although it might work in this simplified example), since further specifics will come into play (making even further segmentation necessary and so on). There is, however, another reason why working with aggregated data will not work in general. Remember that one of our main assumptions was that claims happen on January 1st (which is of course far from reality). If we loosen this assumption, the development years will contain different "parts" of claims cashflows (since they all have different "starting points"), affecting chain-ladder factors accordingly (in practice this is a dominant effect; further shifts of cashflows might be due to delayed or accelerated claim handling, e.g. payments). The clustering into development years requires a somewhat homogenous distribution of claims and payments during the accident year as well as homogenous claims handling over time, which is usually not the case. Analogous examples (to the one considered) might be constructed based on this observation.

This all seems, of course, rather technical, but what does it actually mean for actuarial work in practice? We shall discuss some concrete examples, showing how hard it is to use aggregated reserving results in subsequent processes (such as pricing, risk management, claims steering, etc.).

I Aggregated data and methods make it hard to find out what is really going on in your portfolio (e.g. effects like inflation, portfolio changes, etc. will remain completely in the dark). The volatility in a claims triangle (or its development factors) is a superposition of completely different effects. For instance, what might look like inflation in a claims triangle might actually just be a shift to costlier claims 
types (which is not necessarily a problem, provided an adequate premium is paid). Hence, actuaries will not be able to answer the question, what the main risk drivers of their portfolios are, how they might be quantified and-most importantly-how they might develop in the future. Moreover, it is completely unclear how aggregated methods project effects like inflation or portfolio changes (which are part of the data that constitute claims triangles) into the future.

II In our example, the average case reserve of claims handlers (according to Handelsgesetzbuch, German accounting framework) for the accident year 2019 will be significantly lower than usual due to the fact that the share of smaller claims is larger than usual (claims handlers - to whom detailed claims data is available - may of course include this in their case reserves). Hence, if an actuary were to calculate the best estimate reserve using the chain-ladder method on paid triangles (averaging of past years), their results may seem to contradict case reserves (which does often happen in practice). As a result, actuaries will be forced to make further analysis to resolve this problem. However, without detailed data, this will usually be an impossible task. Depending on the depth of data available, one might find some explaining aspects but usually not get the full picture and a substantial improvement of calculations will be hard to achieve. Especially under the time constraints of Solvency II, this puts actuaries in a difficult position.

III One could argue, that by also considering incurred triangles, this problem would not arise and further insights might be possible. However, working with incurred triangles turns out to be quite a challenge in practice. If the structure of different claims types (Table 1) is adequately and consistently (!) incorporated in case reserves by claims handlers (over time), one could obviously get some benefit from incurred triangles (the problems of superposition of different components will of course remain). This, however, will almost never be the case in practice. Case reserves are heavily biased by the training and experience of claims handlers. Small events like legal changes, changes in claims handling processes or negative experiences (in claims handling) might affect case reserves strongly and, hence, distort incurred triangles to the degree of complete uselessness. Working with incurred triangles requires a very detailed understanding of claims handling, the legal and company-specific background behind reserving as well as the occurred changes over time (in Sect. 3.2 we will discuss these aspects in detail).

In practice, one might also take further information into account such as the claims handling velocity or paid/incurred ratios. Such figures may give an additional hint as to what the correct development factor might be. However, our practical experience shows, that (although this might be helpful in some cases) in general one cannot obtain results which are significantly better.

IV Standard reserving methods make projections for the future entirely based on data of the past. In this setting, incorporating future changes is usually very difficult, if not impossible. Imagine the following scenario: Due to legal changes (court decisions), compensations for personal suffering for heavy injuries (Type III) must be increased by $50 \%$ (in the future). Implementing this adequately using just 
aggregated methods and aggregated data is almost impossible. When modelling all claim types individually (on the level of Individual claims), however, future changes of this kind can easily be incorporated.

V Using aggregated results at the operational level is very difficult, if not impossible. Imagine the following use-case: To aid the recovery of claimants with heavy injuries (Type III) one might consider special treatments for such cases. This will of course increase the cost for medical treatment but might reduce damages for pain and suffering as well as loss of earnings in case consequences are reduced to Type II (or even Type I), say. This is a standard idea in claims management for bodily injury claims (claims steering) and might create a win-win-situation for claimant and insurer. However, in practice it is difficult to evaluate whether such approaches are actually successful. Claims might change their type during claims development (for instance due to better and unexpected healing in case of a young claimant), hence making it hard to understand whether an improvement is really the result of a more intensive treatment. Only an analysis on the level of individual claims might yield a better understanding.

All these points might be addressed by modelling on the level of individual claims. Our example at the beginning shows this quite clearly:

I The volatility in our claims data simply arises from varying shares of claim types. This is the main driver of our portfolio, so we know exactly what is going on.

II Knowing the shares of different claim types (Fig. 1) and understanding their characteristics (as given in Table 1), we can easily calculate the best estimate reserve of our portfolio. It would be absolutely consistent with the case reserves by claims handlers.

III The mentioned changes in damages for pain and suffering can easily be incorporated.

IV The reduction in earning capacity for each claims type gives an adequate benchmark for comparing the effects of different medical treatments. The main question will be, whether the reduction in earning capacity is significantly reduced after certain medical treatments (compared to cases with "standard" treatment of the same claims type). An adequate approach on the level of individual claims might be given by modelling the risk percentages (see example, Type I: 0.2 , Type II: 0.5, Type III: 0.9$)^{2}$.

All this shows clearly that individual claims models can form a common basis for all actuarial processes. Even aspects like IBNR provisions might fit well into this context: The number of IBNR claims might be obtained by a flat-rate model and claims amounts by using individual claims models based on "average" attributes.

\footnotetext{
2 In practice, this turns out to be a very useful approach, esp. since it makes models wage independent.
} 
Moreover, since best estimate reserves are used as a pivotal input in numerous other processes (Asset-Liability-Management, internal modelling, accounting, etc.), a substantial improvement in quality and detailing would also yield substantial further benefits in other areas (as for example shown by the last point above concerning claims steering), reaching far beyond the actuarial department. We strongly believe that it should be the goal of actuaries to actively engage with relevant processes outside the actuarial department and try to support and shape them (using results and insights from individual claims modelling).

However, as said before, due to a lack of detailed data, individual claims models are hard to achieve in practice. However, the effort needed to obtain the required data is completely outweighed by its benefits and definitely worth the while in our opinion.

The following pivotal question remains open: What exact data is needed for individual claims modelling? From our experience, this very essential aspect is unfortunately often neglected. One can never make up for a lack in data by using more sophisticated mathematical models. Our example shows quite clearly that only by having detailed claims data (payment data for each compensation component as well as claim types) one can expect to develop "good" individual claims models (for each component individually!).

In the following section our focus will be on deriving exactly the kind of data needed for developing individual claims models for bodily injury claims in German motor liability insurance. To this end, analysing the legal background of bodily injury claims is a very essential first step. For further aspects on how the required data might be collected or how claims systems should be adapted (so claims handlers can enter relevant data directly and make it available for actuaries), see Amberger et al. (2018) and John and Wiedemann (2018). Although a significant initial effort is needed, collecting relevant additional information in claims systems could yield substantial benefits for claims handling processes as well. The main attributes for individual claims models will be of relevance for claims handlers, so having then available in the system they work with bears significant opportunities (for instance for claims steering etc.).

\section{In detail: claims handling processes and relevant data for individual claims models}

As seen in the last section, actuarial reserving based on aggregated data comes with a number of problems, making it difficult for actuaries to meet the challenges they are currently facing. We believe that the only way forward is to rethink actuarial methods and processes in order to make their results really available at all levels of insurance companies. This, of course, means that they need to be detailed enough so they can be used directly avoiding further modification. From the example in the last section, it became clear, that modelling provisions on the level of individual claims could solve most of the problems straight away. This approach is well established in pricing but currently not widely used in reserving due to a lack of detailed claims data. Nevertheless, in the last years individual claims models have slowly come into 
fashion amongst actuaries, and we believe that they are the only way forward and not just a passing trend.

As mentioned before, developing individual claims models is a lot more than just developing mathematical models. Individual claims models aim to model claims development in great detail and a crucial part in claims development is played by claims handlers. Hence, in a sense, modelling individual claims involves not only modelling the specific characteristics of certain claim types but also the companyspecific behaviour of claims handlers as well. We believe that the best way to achieve this is by establishing a strong cooperation and collaboration between actuarial and claims departments (for further details see Amberger et al. 2018; John and Wiedemann 2018). Thus, the development of individual claims models comes with a new approach to the way actuarial and claims departments should work: They need to work closely together to combine actuarial knowledge of reserving with the experience of operative claims handling. In the combination of both lies a great potential for insurance companies. For actuaries this means, that they are required to have a sound understanding of the (legal) background of claims development and compensation (allowing for a communication basis with claims handlers). In a sense, actuaries need to undergo a (light) training as claims handlers (for details see Amberger et al. 2018; John and Wiedemann 2018). From our experience this will provide them with a solid basis for the development of individual claims models.

In the remainder of this section, we aim to discuss claims handling processes and the legal background focusing on bodily injury claims in German motor liability insurance. Most of the abstract procedural aspects are based on our practical experience but will — as we believe-hold true in general; legal aspects are, of course, specific to each line of business and country.

\subsection{Claims handling_procedural aspects}

It has always been important for actuaries to have a good understanding of the data they work with. Individual claims models will, however, require an even deeper understanding of the data used for modelling (since many more attributes will come into play). A sound understanding of the procedural aspects of claims handling as well as the technical implementation (in claims systems) is absolutely necessary for this (since they are the source of actuarial data). This is, of course, independent of the portfolio or legal framework.

In the following, we shall focus on the main procedural aspects of claims handling for German non-life portfolios. For other portfolios or legal frameworks, a completely similar analysis will yield a sound basis for individual claims models. Since the technical implementation will be company-specific (depending on claims system used), we shall not discuss these aspects.

Once a claim is reported, there are basically two steps:

- Initial assessment: After notification of a claim, claims handlers collect relevant information/attributes and set an initial case reserve (based on initial informa- 
tion). As required by the German Commercial Code (Handelsgesetzbuch ${ }^{3}$, esp. $\S 252 H G B$ and $\$ 341 e-f H G B)$, case reserves must be set individually per claim taking into account the specifics of this claim. Moreover, they must be prudent and safe (contain an implicit safety margin). In practice, case reserves will be based on individual experience as well as company policy and vary heavily between claims handlers. From an actuarial point of view, case reserves might be thought of as a composition of a best estimate reserve together with a risk margin. Components are, however, not separable as they are not assessed individually by claims handlers but as a whole.

The key aspect is the term "relevant information/attributes", by which we mean all factors that determine the development and characteristics of a claim (used by claims handlers to assess and handle claims). In case of bodily injury claims, such factors might be for example injury, reduction in earning capacity, age, wage, occupation of claimant, etc. (for further details see next section). It is important to understand, that not all information will be known right after claims notification and in some cases, it might take months for it to be available. In case of severe bodily injuries for instance, it might take a very long time (due to hospitalisation) to get in touch with the claimant and obtain relevant information (in such cases a legal representative or next of kin might be able to provide information at an earlier stage). In those situations, claims handlers need to set case reserves based on the information they have as well as their experience (at very early stages, with not much information known, only rough estimations might be possible). As more information becomes available, estimates might improve in detail and quality. Moreover, important factors like injury or reduction in earning capacity might change considerably over time as the outcome of an accident becomes clearer.

Consider the following example of a claimant with an injured leg and arm. Right after notification, it might not be clear which injury will cause most problems later on. One of both might seem heavier right after notification, but due to a healing process far better than expected, later on the other injury might have more severe consequences.

For modelling individual claims it is of utmost importance to understand these specific aspects. Moreover, it is also important to understand how claims systems fit into claims handling, since they are the source of all data used for individual claims modelling (and an interpretation of data requires an understanding of how it is generated). For instance, actuaries need to understand the following questions: What claims information is collected in claims systems? When (during claims handling) are relevant attributes entered into the system (after notification as a possible estimate or only when definitely known)? This again might vary between claims handlers.

- Subsequent claims handling: Claims handlers update relevant information/ attributes, make payments, negotiate (lump sum) settlements and update case reserves appropriately. These actions will continue until the claim is settled.

\footnotetext{
3 German accounting framework.
} 
Subsequent claims handling actions will be triggered by different events such as:

a. Contact with parties involved (policy holder, claimant, legal representative, next of kin, doctor, police, etc.): As a result, relevant information/attributes (injury, reduction in earning capacity, wage, etc.) might be updated and (if necessary) a re-evaluation of case reserves will be triggered. The information known at certain stages during claims handling will strongly depend on the approach by claims handlers (they might for instance actively approach parties involved for further information or wait passively).

b. Payments to different parties:

i. Regular invoices (hospital, health insurance body, claimant, etc.): Triggered mostly externally, as a result case reserves will usually be re-evaluated.

ii. Settlements of compensation component (with claimant, health insurance body, etc.): Triggered by claims handlers or external party, as a result concerned compensation component will usually be (partially) settled. If future risks are excluded from settlements, appropriate case reserves will be set. From an actuarial point of view, these risks will usually be very hard to model due to a lack of data. As already mentioned above, the approach of settlements by claims handlers will have a huge effect on the development of claims. By actively approaching parties involved for settlements, claims might be settled significantly faster than by waiting passively. In this context, company policy as well as behaviour of external parties will also play an important role and significant changes over time will obviously have a huge impact on claims cashflows (usually rendering aggregated methods inapplicable).

From an actuarial point of view, it is important to understand that claims handlers will normally pay the amount owed directly to the party concerned (which is usually also the amount entered into the claims system). This amount, however, might be a sum of payments for different compensation components (for instance an invoice from a health insurance body might contain payments for medical treatment, sick pay, etc.). For individual claims modelling, payments for each compensation component are needed which might in practice be a huge hurdle since it requires adapting claims systems (and claims handling processes) to collect payment data in greater detail.

c. Regular reviews: Internal control systems will usually require regular reviews of claims to avoid undiscovered risks or negative developments (review intervals will usually depend on claims sizes). This will only be relevant for claims without regular transactions (e.g. payments, etc.), which have not been settled (for instance, due to possible future care costs, a claim might not be settled although currently there are no expenses for care costs). Claims handlers will mainly try to update relevant information/attributes by contacting parties involved (claimant, health insurance body, etc.) and possibly reassess case reserves in case of significant changes (severity of injury, death of claimant, etc.). 


\subsection{Bodily injury claims-legal background}

The aim of (monetary) compensation is to put claimants back in a position as if the accident had not happened. However, assessing the consequences of an accident monetarily is usually quite difficult and one of the main challenges of claims handling. In this process, claimants will play a crucial role. They will usually have a strong interest themselves to reduce the consequences of the accident in order to continue their day-to-day life's with as little restriction as possible (which is unfortunately not always possible, esp. in case of heavy accidents with severe injuries). Moreover, claimants are obligated ( $\$ 254 \mathrm{Abs.} 2 \mathrm{BGB}^{4}$ ) to take all reasonable actions to avert or reduce damages 5 . In certain cases a joint liability of the claimant might be considered, reducing claims compensations accordingly ${ }^{6}$.

For the development of individual claims models, it is very important to have a sound understanding of the legal background of claims. Based on this, one can derive relevant information/attributes for individual claims models. Analysing claims handling processes will of course already reveal many relevant aspects. However, for detailed modelling it is important to get a deeper understanding as to why certain aspects are relevant and how they have changed in the past or might change in the future.

In the following we shall discuss the legal background for claims handling of bodily injury claims for German motor liability portfolios. Our elaborations are mainly based on Küppersbusch and Höher (2016) (a very detailed and comprehensive source on this matter). Our aim is not to give a comprehensive overview but a brief outline of the account given in Küppersbusch and Höher (2016) (of the main characteristics of all claims components), especially focussing on the relevant aspects for non-life actuaries. For further details such as references to relevant legal texts and court rulings, we refer the reader directly to Küppersbusch and Höher (2016).

Our main focus will be to determine relevant attributes for individual claims models. In doing so, we will take a pragmatic approach and only focus on attributes which can be obtained without disproportionate effort. In practice, not all of these attributes will be maintained in claims systems and therefore not be available for actuarial modelling. We believe, however, that since the aspects described are very essential for claims handling, implementing them into claims systems would be very beneficial not only for the development of individual claims models but also for claims handlers and claims handling processes. We shall also discuss how inflation might be assessed for each compensation component in order to adjust data from different reporting years appropriately to obtain a homogeneous basis for modelling.

\footnotetext{
4 German Civil Code (Bürgerliches Gesetzbuch).

5 In particular, claimants must undergo medical treatment if it is promising and safe (in case of surgery claimants are only obligated, if it is harmless, riskless with promising outcome and substantial improvement-which is almost impossible to prove in practice). Moreover, claimants must utilize any remaining ability to work within reason to reduce loss of earnings damages or maintenance damages.

6 Under certain conditions, driving under the influence of alcohol or not wearing safety helmets or safety belts might give rise to joint liability.
} 
Moreover, we shall discuss the parties involved (claimant, health insurance body, pension insurance body, etc.) for each claim component, which is very crucial for understanding the cashflows for each component (since each party involved will follow different procedures for claiming payments).

\subsubsection{Pain and suffering}

Damages for pain and suffering are awarded for injuries of body or health, which are a direct result of the accident event ( $\$ 253$ Abs. 2 BGB). Before 2002, compensation for pain and suffering was only owed in case of liability of injuring party. Since 2002 strict liability (Gefährdungshaftung) also gives rise to pain and suffering claims (hence, damages can be claimed even in case injuring party is not liable).

The aim of pain and suffering damages is twofold: compensation (for non-financial losses) and satisfaction (to make up for what was done by the injuring party). Normally, compensation outweighs satisfaction (especially in car accidents). Since 2017, close relatives can also claim pain and suffering damages (Hinterbliebenengeld, see Deutscher Bundestag 2017) in case of death of an accident victim (without having to prove an impairment $)^{7}$. Before, close relatives could only claim damages in case they suffered from impairments beyond an expected mourning reaction of significant duration as a consequence of the accident event (Schockschaden).

Joint liability of the claimant will affect the satisfaction component of damages for pain and suffering. However, the Federal Supreme Court ruled that a mere mathematical quotation of damages is not permitted (since joint liability is only one factor for assessment among many others). In practice, however, courts will usually not differ much from quoted damages in case of joint liability.

In order to evaluate pain and suffering damages, the intensity and duration of pain and suffering, disfigurement and psychological impact needs to be considered. The following aspects might give an orientation:

- Type of injury

- Number and severity of operations

- Duration of treatment

- Duration of incapacitation for work

- Reduction in earning capacity (Minderung der Erwerbsfähigkeit, $M d E)^{9}$

- Psychological consequences (impact on hobbies, choice of profession, etc.)

Furthermore, age will play a role in connection with the expected duration of suffering and awareness (which will be shorter, the older claimants are) $)^{10}$. Further aspects like a poor physical constitution prior to the event or a short period of

\footnotetext{
7 Compensations between 10,000 and $20,000 €$ are sometimes mentioned in relevant sources (see for instance Deutscher Bundestag 2017).

8 Moreover, damages for pain and suffering of claimants are hereditary.

9 Percentage figure describing the (general) impairment of earning capacity as a result of the accident event.

10 There are, however, only very few legal decisions on this matter.
} 
survival after the event will also be taken into account (both might usually reduce damages).

When determining damages, claims handlers and courts will normally use standardised tables (so-called Schmerzensgeldtabellen), which record the facts and amounts awarded in previous cases ${ }^{11}$. However, they may still decide to take specific aspects of individual cases into account and, hence, award amounts of a different magnitude. Moreover, when referring to similar previous cases, inflation might be taken into account. For instance, in Hacks et al. (2021) amounts are adjusted using the Consumer Price Index (CPI). Hence, CPI might give an indication for inflation of pain and suffering damages.

Damages for pain and suffering will usually be settled by lump sum payments, annuity settlements being an exception (for instance in case of severe injuries with enduring pain). The present value of annuity settlements together with a lump sum settlement must be roughly equal to lump sum settlements in similar cases.

From an actuarial point of view the following attributes seem relevant for modelling damages for pain and suffering:

- Type of injury and severity

- Reduction in earning capacity

- Age of claimant

From our experience, all the other criteria given above do not seem to be available for modelling without disproportionate effort nor necessary for developing good models.

When using injury types and injury severities for modelling, one will obviously need a sufficient clustering defining homogeneous (and sufficiently large) subgroups. To this end, one might use ICD-Codes ${ }^{12}$ or the so-called Weller-Database ${ }^{13}$ of injuries.

In practice, however, the main hurdle will be the correct assignment of codes, which usually requires advanced medical knowledge and is thus not straightforward. Moreover, normally there will not be a single well-defined injury but (multiple) primary and secondary injuries, requiring an appropriate ranking (which may change over time).

\subsubsection{Medical treatment}

Reasonable medical costs (medical treatments and care, medicine, physiotherapy, cosmetic surgery, etc.) for bodily injuries caused by the accident event are to be reimbursed (it is important to stress, that suspicion of injuries will not suffice for

\footnotetext{
11 Two of the most well-known tables are Hacks et al. (2021) and Slizyk (2022).

12 International Statistical Classification of Diseases and Related Health Problems (ICD).

13 Coding used by employers liability insurance bodies (Berufsgenossenschaften), classifying injuries. It has the advantage of also allowing for the classification of severity given sufficient medical knowledge. See for instance https://www.fsa.de/produkte/steuerung-des-heilverfahrens-weller-datenbank/ [last downloaded 27th November 2019].
} 
claiming compensation). Additional expenses (for instance telephone charges, travel expenses ${ }^{14}$, etc.) will also be compensated ${ }^{15}$.

In practice, compensation will mainly be based on the coverage of the respective health insurance scheme. The main coverage schemes are:

\section{Statutory health insurance (Gesetzliche Krankenversicherung $)^{16}($ see Wikipedia} 2021)

2. Employers liability insurance, accident insurance ${ }^{17}$ (Berufsgenossenschaften, Unfallversicherung) ${ }^{18}$ (see Wikipedia 2021)

3. Private health insurance (Private Krankenversicherung)

4. Insurance schemes for civil servants (Beihilfe)

Bodily injuries will usually require hospital treatment initially and perhaps later on (unless injuries are only minor), constituting a large share of the overall medical treatment costs. Since 2004, the remuneration of inpatient treatment in Germany is based on a DRG system ${ }^{19}$ (Diagnosis Related Groups, flat rate system) with standardised base rates and weighting given by $\mathrm{DRGs}^{20}$ (see Bundesgesundheitsministerium 2021). Statutory health insurance, private health insurance and insurance schemes for civil servants are based on the DRG system, although the scope of the latter two will usually be more extensive (for instance treatment by senior physician or single room accomodation).

Specialised hospitals (treating severe injuries) can diverge from the DRG system and agree separate remuneration contracts with insurance schemes. This is mainly the case for employers liability insurance (Berufsgenossenschaften) and accident insurance (Unfallkassen), which provide a wider range coverage. The reason is that these schemes are not just aiming to restore physical health but also to restore performance and ability to work of casualties with all suitable means to enable lifelong participation in professional life. As a result, inpatient treatment and especially rehabilitation will be more extensive compared to statutory health insurance for instance. Moreover, policyholders are entitled to a life-long aftercare.

Note that claimants are not necessarily bound by the scope of their coverage (for instance alternative treatments or therapies are to be reimbursed if not covered but suitable). Under some circumstances, claimants with statutory health insurance might also be entitled to the benefits of private health insurance coverage

\footnotetext{
14 Including expenses incurred by close relatives.

15 Possible savings (for instance board is provided during stays in hospital) will, however, reduce compensation (usually taken into account when compensating damages for loss of earnings).

16 Social Security Statute Book V (SGB V, Sozialgesetzbuch Fünftes Buch).

17 Covering work-related accidents, for instance accidents occurring during commute to or from work.

18 Social Security Statute Book VII (SGB VII, Sozialgesetzbuch Siebtes Buch).

19 The introduction of the DRG system in Germany started in 2003 on a voluntary basis. Since 2004 the DRG system is compulsory.

20 Starting point were different base rates for each German federal state. Between 2005 and 2009 base rates of hospitals converged towards the base rate of the corresponding federal state. Between 2010 and 2014 the base rates of federal states converged towards a Germany-wide base rate corridor.
} 
(for instance in case of additional private insurance or by choosing the so-called Kostenerstattungsverfahren).

In Germany almost everyone has health insurance coverage. In order to avoid double compensation, claims for which the claimant might be entitled to social benefits (esp. covering medical treatment costs) will be transferred to the respective social security body (health insurance body) by law at the event of a claim ${ }^{21}$ (hence, claimants will usually never be owners of the respective claims). As a result, handling medical treatment costs will take place mainly between the insurer and the corresponding health insurance scheme of the claimant (which will claim all costs incurred $)^{22}$, hence adding another important player to claims handling.

Medical treatment costs will usually be compensated as incurred by the respective health insurance body or claimant. Lump sum settlements (once course and consequences of the injuries are known or safely predictable) are also possible.

Inflation of medical treatment costs for bodily injury claims will mainly depend on the scheme of coverage. A large share is constituted by hospital treatment costs. It is-apart from Berufsgenossenschaften and Unfallkassen-linked to the development of Germany-wide base rates over time ${ }^{23}$. Other medical treatments (mainly outpatient treatment by general practitioners, specialists, etc.) will also depend on the scheme of coverage ${ }^{24}$.

From an actuarial point of view the following attributes seem relevant for modelling medical treatment:

- Type of injury and severity ${ }^{25}$

- Type of health coverage scheme

- Age of claimant

The first two attributes seem obvious. Age of claimant might be relevant on the grounds that the consequences of accident events might be less severe for younger claimants due to better physical conditions.

As mentioned before, getting consistent data on injuries and severities might prove difficult in practice (esp. severities require detailed medical knowledge). A rough proxy for the severity of an injury might be given by the reduction in earning capacity

\footnotetext{
$21 \S 116$ Social Security Statute Book X (SGB X, Sozialgesetzbuch Zehntes Buch).

22 If claimants are entitled to further benefits (not covered by their health insurance scheme), they will need to claim these directly. In rare cases with no insurance coverage, claimants will need to claim all medical costs themselves.

23 Germany-wide base rates are negotiated between the German Hospital Association (Deutsche Krankenhausgesellschaft, $D K G)$, Statutory Health Insurance Association (GKV-Spitzenverband) and Private Health Insurance Association (Verband der Privaten Krankenversicherung, PKV). The base rate for 2019 amounted to $3544.94 €$. Base rates of federal states must be within a corridor of $-1.02 \%$ and $2.50 \%$ of the Germany-wide base rate.

24 Remuneration of medical treatment by contracted doctors (Vertragsärzte, statutory health insurance) is regulated by the Social Security Statute Book V (SGB V, Sozialgesetzbuch Fünftes Buch) based on a valuation standard (Einheitlicher Bewertungsmaßstab). Remuneration of medical treatment by non-contracted doctors is regulated by the German Scale of Medical Fees (Gebührenordnung für Ärzte).

25 Here, the same remarks made earlier for clustering types of injuries apply.
} 
(Minderung der Erwerbsfähigkeit, $M d E$ ), since severity of injuries and impairment resulting from the accident event seem linked (there are, of course, exceptions).

\subsubsection{Loss of earnings}

Damages for loss of earnings comprise loss of income as well as all negative economic impacts resulting from an impairment of the ability to work due to the accident event. Sole impairment of the ability to work will, however, not suffice (there must be a specific pecuniary $\operatorname{loss}^{26}$ ).

Damages comprise for instance lost salaries (including certain bonuses) of employees or lost profits of self-employed. Moreover, impairment in housekeeping (such as repair work, etc.) ${ }^{27}$ concerning family support ${ }^{28}$ as well as parenting also constitute a loss in earnings ${ }^{29}$, since partners are obligated to housekeeping support. Possible benefits (such as non-incurring work-related expenses) will be deducted.

To reduce damages (as described earlier) claimants must utilise their remaining ability to work (if reasonable and possible) ${ }^{30}$. Aspects such as state of health, personality, social position, education, family, residence, past work life, etc. will be taken into account when judging reasonability. In case continued employment with the current employer is not possible, claimants must search for a new employer. Possible costs for retraining, vocational reintegration, modification to workplace, etc. are to be reimbursed if reasonable ${ }^{31}$.

A loss in earnings will usually entail a reduction in pensions as well (due to lower pension contributions). The corresponding claim is transferred to the respective pension insurance body by law at the event of a claim ${ }^{32}$. As a result, pension insurance bodies are important players when it comes to settling loss of earnings claims, since they act as trustees claiming reductions in pensions (so claimants are entitled to pension levels as if the accident event had not happened).

Damages for loss of earnings are determined on the basis of a forecast of the professional career as well as the development of salaries or lost profits of claimants assuming the accident event had not happened. However, such a forecast must be sufficiently likely and cannot be completely fictitious. For this, concrete indication based on the life of the claimant (before and after the accident event) will be taken into account. In case of claimants with steady working life, such a forecast will not

\footnotetext{
${ }^{26}$ For instance, due to interruption of current working activity or limited prospect of future working activity (generating actual earnings).

27 Claims must not be excessive but more than just small assistances in housekeeping. Efforts must be feasible and non-postponable (after recovery).

28 Impairments concerning personal needs constitute damages for additional needs.

29 Singles are also entitled damages for housekeeping, however there are restrictions since delaying housekeeping work may be much easier than in the case of families.

30 Any generated income will reduce damages for loss of earnings.

31 Such measures will usually be carried out by social security bodies but might also be carried out by the insurance company handling the claim.

$32 \S 116$ Social Security Statute Book X (SGB X, Sozialgesetzbuch Zehntes Buch).
} 
be too difficult ${ }^{33}$. In all other cases, however, any forecast will contain a higher level of uncertainty. Forecasts are particularly difficult for the following groups

- self-employed, craftsmen, freelancer ${ }^{34}$

- unemployed ${ }^{35}$

- children, pupils, apprentices, or students ${ }^{36}$.

Housekeeping damages are assessed on the basis of tasks that cannot be fully carried out due to impairment caused by the accident event. For this, one might estimate the time needed for respective tasks (for instance by using standardised tables, see for instance Pardey 2021), the impairment ${ }^{37}$ (in housekeeping ${ }^{38}$ ) resulting from the accident event as well as the cost for a substitute. Due to diminishing body strength, annuity damages might be restricted to the end-age of 75 (assuming that assistance would be needed after this point anyway).

Handling loss of earnings claims will involve several claimants. For instance, in case of employees, employers ${ }^{39}$ might claim continued payment of wages (for a maximal period of 6 weeks following the accident event) and health insurance bodies might claim sick pay (Krankengeld, Verletztengeld, etc. ${ }^{40}$ as well as health insurance contributions (for a maximal period of 18 months after the accident event) ${ }^{41}$. Moreover, employers liability insurance and accident insurance schemes (Berufsgenossenschaften, Unfallversicherung) will—as already mentioned above-offer more extensive treatments (esp. rehabilitation) for which claimants are entitled to sick pay. The corresponding periods will usually be longer compared to statutory health insurance coverage (with sick pay being usually higher as well). In case of private health insurance, the situation will depend on the individual coverage chosen.

As a result (depending on the situation of the accident victim), claims handling can become quite involved with several claimants and numerous regulations to be

\footnotetext{
33 One might refer to a similar person (for instance a colleague) to determine the development of salaries or profits as well as the professional career. But even then, there is still the possibility of unemployment later on (for instance in case of bankruptcy of employer).

${ }^{34}$ Forecasting business development is usually very difficult. In such cases one might try to estimate the reduction in profits due to the accident event.

35 Simply assuming that the current unemployment situation will continue in the future is not valid, for claimants might have gotten back into work without the consequences resulting from the accident event.

36 Forecasts might include aspects such as family background (professional career of parents or siblings, etc.) personal aptitudes and academic achievements. Damages must take into account facts like additional income (for instance in case of part-time jobs), reduced income due to delayed start of career as well as delayed rise in income.

37 Small impairments will usually not constitute significant restrictions. In such cases, one might for instance compensate an impairment by a re-organisation of tasks or by accepting an insignificant increase in time needed.

38 There are tables available (see for instance Pardey 2021) containing the impairment in housekeeping (Minderung der Haushaltsfiuhrung) for different injuries (as percentage figures).

39 Corresponding claims will be transferred to the employer at the event of a claim.

40 Possible tax privileges (sick pay is for instance tax-exempt) will reduce damages.

41 As described above, the corresponding claims will be transferred to the respective body at the event of the accident by law.
} 
taken into account. For further details we refer the reader directly to Küppersbusch and Höher (2016).

From an actuarial point of view, it seems reasonable to model housekeeping damages separately from the remaining aspects of loss of earnings, since it will depend on different attributes (for instance on the family situation of claimants). This will of course require the corresponding data to be available. We thus propose the following subdivisions of damages for loss of earnings:

- Damages for housekeeping

- Remaining damages for loss of earnings (not including housekeeping)

The following attributes seem relevant for modelling housekeeping damages:

- Family situation of claimant (single, married, etc.)

- Age of claimant

- Impairment in housekeeping (Minderung der Haushaltsführung, $M d H$ )

In case impairment in housekeeping is not available, one might use reduction in earning capacity $(M d E)$ as a rough proxy. However, it is important to understand, that both figures describe (very) different aspects. Hence, reduction in earning capacity might just give a rough indication.

The following attributes seem relevant for modelling remaining damages for loss of earnings (not including housekeeping):

- Reduction in earning capacity

- Type of health coverage scheme

- Age of claimant

- Occupation and wage of claimant (including forecast of future)

Particularly difficult seem to be future forecasts of occupation and wage. Claims handlers will have to make assumptions depending on the overall view they have of the claimants' life situation. This will depend on many soft aspects, which are impossible to collect systematically in claims systems and, hence, cannot be used for modelling. Modelling seems only possible on the basis of coarse information of (future) occupation and wage.

In our experience, a reasonable clustering of occupational groups will suffice. First of all, a separation between physical work and non-physical work is very important (especially separating workers and employees), since claimants performing physical work will be more affected by physical injuries, resulting in a higher risk that damages for loss of earnings will be claimed. Moreover, groups like civil servants and self-employed will vary in damages due to the numerous specifics of both groups (sick pay, pension, etc.).

Inflation in both components (housekeeping and remaining damages) will strongly depend on the inflation of wages and salaries. 


\subsubsection{Additional needs}

Damages for additional needs comprise all recurring and concretely ${ }^{42}$ accrued expenses aiming to compensate for disadvantages due to the impairment of physical well-being caused by the accident event ${ }^{43}$.

Examples of expenses to be reimbursed are: care costs, costs for support to attend schools or workshops for disabled, disability vehicle, orthopaedic aids, wheel chairs, barrier-free renovations, etc.

Damages for additional needs are usually settled as annuity payments (exception one-off payments such as cost for home/car conversion, etc.). However, lump sum settlements (if agreed with claimant) are also possible.

Of particular interest are care costs, since cases requiring (possibly intensive) care (as a result of the accident event) are potentially very expensive and very hard to predict. Care costs comprise: care homes, hiring nursing staff (in case of care at home), expenses of family members (in case they carry out care), etc.

In Germany almost everyone has nursing care coverage, since health insurance bodies are required to offer their policyholders nursing care insurance ${ }^{44}$ (see for instance Wikipedia 2021) as well. Depending on the type of insurance, coverage will be more or less extensive. Moreover, employers liability insurance and accident insurance will provide nursing care in case of work-related accident events (with coverage being usually more extensive as in the case of statutory nursing insurance). Completely analogously to medical treatment costs, claims will be transferred to the respective nursing care body at the event of a claim, bringing further claimants into play.

Payments of nursing care bodies are linked to so-called care degrees ${ }^{45}$ (Pflegegrade (see for instance Bundesgesundheitsministerium 2021)) describing the extent of care needed with different amounts for each degree (also varying between outpatient or inpatient care). If actual care costs are higher than the payments for the assigned care degree, claimants need to claim additional amounts directly.

Inflation of nursing care costs will mainly depend on the scheme of coverage. It is linked to the development of compensation amounts for different care-degrees over time.

From an actuarial point of view, it seems reasonable to separate nursing care costs from the remaining aspects of additional needs and model them separately, since the magnitude of nursing care costs can be quite substantial. This will of course require the corresponding data to be available. For modelling, we thus propose the following subdivisions of damages for additional needs:

\footnotetext{
${ }^{42}$ Fictitious expenses are not to be reimbursed. Non-material disadvantages form part of pain and suffering damages.

${ }^{43}$ Not included are expenses for medical treatment (which aim to restore physical health) and general living expenses.

${ }^{44}$ Social Security Statute Book VII \& XI (SGB VII \& XI, Sozialgesetzbuch Siebtes \& Elftes Buch), analogous regulations in case of private care insurance.

45 Introduced in 2017 (before 2017 so-called Pflegestufen) with 5 different care degrees (Pflegegrad 1-5). Care degrees are assigned by assessment through a medical service (Medizinischer Dienst) or a similar expert service.
} 
- Nursing care costs

- Remaining additional needs (not including nursing care costs)

For modelling nursing care costs, the following attributes seem relevant:

- Type of injury and severity

- Type of care coverage scheme and care degree

- Care situation of claimant (outpatient, inpatient)

- Age of claimant

Since payments of nursing insurance providers are linked to care degrees, these attributes are relevant. Moreover, there are different amounts (per care degree) for inpatient and outpatient care. Especially inpatient nursing care and in-home nursing of cases requiring intensive care need to be taken into account, since they might be very costly with care allowances (of care insurances bodies) usually not covering all costs.

Type of injury and severity will only be relevant in cases with severe injuries (for instance paraplegia, severe head injuries, etc.) requiring care to a very high extent. One might consider modelling such cases separately.

For modelling the remaining aspects of additional needs (not including nursing care costs), the following attributes seem relevant:

- Type of injury and severity

- Care situation of claimant (outpatient, inpatient, no nursing care)

- Age of claimant

For cases requiring nursing care, the magnitude of damages for remaining additional needs might also be substantial higher (for instance in case of intensive care at home requiring considerable modification of home and expensive care devices). Hence, remaining additional needs should be modelled differently in cases requiring nursing care.

As mentioned before, a rough proxy for the severity of an injury might be given by the reduction in earning capacity (Minderung der Erwerbsfähigkeit, MdE).

\subsubsection{Maintenance}

The number of traffic fatalities in Germany declined considerably between the beginning of the 1990's and roughly 2010 (with numbers of vehicles increasing significantly at the same time). Since then, traffic fatalities seem to roughly stagnate with $3275^{46}$ fatalities in 2018 . The overall number of bodily injuries (resulting from traffic) in 2018 amounted to roughly 396,00047, showing that maintenance damages will play a minor role in reserving. Moreover, since the assessment of maintenance claims follows rather technical case-dependent rules, modelling seems difficult from an actuarial point of view. For this reason we shall restrict ourselves to presenting just a rough overview.

\footnotetext{
46 Source: German Federal Statistical Office (Statistisches Bundesamt, see www.destatis.de).

47 Source: German Federal Statistical Office (Statistisches Bundesamt, see www.destatis.de).
} 
The killing of an accident victim ${ }^{48}$ (as a result of the accident event ${ }^{49}$ ) with an obligation to provide will entitle dependants ${ }^{50}$ to claim lost maintenance payments (during the supposed lifetime ${ }^{51}$ of the victim assuming the accident had not happened).

Damages are based on legally owed maintenance (not on actual payments made by victim) as well as personal and financial capabilities of the accident victim ${ }^{52}$. Damages comprise all needs of families: cash maintenance (Barunterhalt) as well as non-cash maintenance (personal support and care to which dependants are entitled, Naturalunterhalt) such as housekeeping and parenting ${ }^{53}$.

Cash maintenance (Barunterhalt) is in practice usually based on different maintenance rates (Unterhaltsquoten, percentage rates) for dependants (widow, widower, orphan). Damages are roughly given by the following formula:

(net income-fixed $\operatorname{costs}^{54}$ ) - maintenance rate + fixed costs-pension (of dependant ${ }^{55}$ ) - income (of dependant) ${ }^{56}$ - possible benefits (of dependant) ${ }^{57}$

The assessment follows standardised methods and formulae in most cases (single earner, double earners, etc.), which we shall not discuss in detail. For further details and examples, we refer the reader directly to Küppersbusch and Höher (2016).

Assessment of non-cash maintenance (housekeeping, parenting) depends on how it is accomplished in specific situations. If, for instance, dependants carry out housekeeping by themselves, damages are assessed by comparison with an adequate replacement on the basis of hours needed (see for instance Schulz-Borck and Hofmann 2000) and an hourly rate. In case a substitute is hired, damages will be based on corresponding costs (but only for services owed).

From an actuarial point of view, the following attributes seem relevant for modelling maintenance damages:

- Family situation (single, married, etc.) and number of dependants

- Age of claimant

\footnotetext{
48 Joint liability will be taken into account.

49 Not necessarily immediately after the accident event but as a consequence of it. Further dependants after the accident event are not entitled to maintenance payments.

50 Such as spouses, children, registered partners, etc.

51 Based on statistical life expectancy, lifestyle, health situation, etc. of the accident victim. In some cases, obligation for maintenance might even expand beyond death (for instance for self-employed with obligation to build up reserves).

52 For instance, in case no maintenance payments were made and without any real chance of enforcing them, no claim arises.

53 Division of housekeeping and parenting (of children under 18 years of age) between partners will usually be agreed by consensus. This is also considered to be what is legally owed, allowing for modifications in case of disproportionate imbalance between partners. Dependants are expected to assist in housekeeping with extend depending on social status, family size, household size, etc.

54 Dependants are entitled to fixed costs such as rent, heating costs, water costs, etc.

55 Widow, widower, orphan.

56 Depending on education, capabilities, etc. dependants are required to paid work. The corresponding income is being taken into account.

57 For instance, ending obligation of cash maintenance for deceased spouses.
} 
- Household situation (fixed costs, etc.)

- Occupation and wage of claimant (including forecast of future)

- Occupation and wage of dependants (including forecast of future)

Getting sufficient data for modelling will, however, be a challenging task in practice. Since-as mentioned before-maintenance damages play a minor role in reserving, simple models (for instance based on case reserves set by claims handlers using a rough model to eliminate the implicitly contained risk margin) might be sufficient.

\subsubsection{Burial}

Damages comprise costs for a dignified funeral taking into account the life situation of the deceased claimant.

From an actuarial point of view, a detailed modelling of this component does not seem necessary, since damage amounts are of minor magnitude. For practical purposes, a simple flat rate model should suffice. As in the case of maintenance damages, funeral costs will play a minor role in reserving.

\section{Consequences for non-life actuaries and further aspects}

The last section showed that bodily injury claims (in German non-life insurance) are a rather complex issue. The main reason being the number of different compensation components (with different relevant attributes) as well as the number of parties involved (claimant, health insurance body, pension insurance body, etc.). It is important to stress, that the (long-tail) run-off patterns of all components will differ significantly and will depend heavily on two main factors: the injuries caused by the accident as well as the healing process but also the behaviour of all parties involved (esp. their tendencies towards lump sum settlements). Hence, external effects like car safety, medical progress and general economic development will play an important role.

We also see that our introductory example in Sect. 2 was not artificial but much too simplistic. In this small example we already saw that aggregated (triangle) data (being a superposition of different claim components) was completely distorted and very hard to interpret. Taking all claim components into account, the situation will, of course, be even more delicate and achieving good results based on just aggregated (triangle) data seems a rather difficult task, if not impossible. The step towards individual claims modelling thus seems natural. It has also become very clear, that this cannot be done without very detailed claims data. However, in practice, detailed data will usually not be available and collecting it is a major and very time-consuming task: One needs detailed data on payments as well as on all relevant attributes for all 
compensation components individually ${ }^{58}$. This is the main reason, why individual claims models are so hard to develop and currently not widely used.

Collecting necessary data for modelling might be done by the following approach (for further details see Amberger et al. 2018; John and Wiedemann 2018). Firstly, one might adjust claims systems, so relevant data can be entered by claims handlers directly and is, hence, available for automatic processing. However, it will take a long time to gather sufficient data due to the long-tail character of bodily injury claims. Secondly, one might take a (representative) and sufficiently big sample from the claims portfolio and extract relevant information from it manually (which is not a straightforward task). In this way, one could achieve a data base to get started and bridge the period of adjusting claims systems.

In the last section, we have mainly covered the characteristics of bodily injury claims (in German non-life insurance) from a theoretical point of view as a basis for developing individual claims models. There remain, however, open questions and difficulties which might also give rise to interesting further research.

1. We have only focussed on bodily injury claims in German non-life insurance. In other regulatory frameworks the situation will, of course, be very different and needs to be analysed in the same detail.

2. There will be a strong connection between actuarial reserving and company-specific internal claims handling processes. Hence, models will differ significantly for different companies. Note that traditional reserving methods are also affected by this. Hence, a scientific analysis of the effects of internal processes on actuarial reserving could potentially be very interesting.

3. One of the most important questions is, of course, the following: Given detailed data, how good can models get? Which approach (GLM, Neural Network, Decision Tree, etc.) is best suited for modelling but also for applying individual claims models in practice? Here lies a large field for potential actuarial research.

4. The relevant attributes discussed in Sect. 3.2 focused-based on our experience-on which ones seem absolutely essential and can be obtained without disproportionate effort. There might, however, be additional attributes needed (which might be more difficult to obtain). Moreover, the attributes mentioned might not all be equally relevant, so that-in order to develop sufficiently good models - some might be neglected (hence simplifying the collection of relevant data). While in the context of pricing, relevant attributes are well understood, in the context of reserving they are not. From an actuarial point of view, it would be very interesting to compare different claims portfolios and investigate the effects of the attributes mentioned and, thus, develop a sound understanding of bodily injury claims in the context of actuarial reserving.

5. On the basis of detailed claims data, key aspects for bodily injury claims may be investigated in detail for the first time. The impacts of the following aspects and risk drivers on claims amounts and run-off patterns seem particularly interesting:

\footnotetext{
58 Note that attributes may change over time and (for instance type of injury and severity) and, hence, it might be necessary to also collect information on how attributes changed during the process of claims handling.
} 
a. Legal changes (for example the introduction of the DRG system or reforms of nursing care coverage)

b. Behaviour of parties involved and patterns of lump sum settlements, esp. in connection with the general economic development

c. Medical progress and longevity

d. Inflation

6. As mentioned earlier, injuries (and healing processes) will play a pivotal role for bodily injuries claims and, hence, are very relevant for modelling. However, attributes like type and severity of injury are very hard to collect. It is difficult to reduce complicated injury data to "simple" attributes one can easily work with. First of all, there might not be just one injury but several and, second of all, injuries (and severity) might change significantly during the run-off period. Moreover, most times it will take a while until injuries become apparent. Suitable approaches to model injuries are yet to be developed, they might form the basis of individual claims models.

7. As we have discussed many times, detailed data is the key for developing individual claims models. However, the total number of bodily injury claims is-compared to all motor liability claims-rather small. Hence, in order to develop individual claims models for bodily injury claims, one needs a motor liability portfolio which is sufficiently large to obtain a data base which is big enough for modelling. However, even then-due to the complexity of bodily injuries claims - there might still not be enough data for all individual attributes. It will for instance be very hard, to get sufficient data to model all types of injuries sufficiently well. This also has a huge impact on the mathematical models used: For (sub-)segments with insufficient data for modelling, expert judgement will play an important role for model calibration (requiring a deep understanding of claims characteristics). Hence, models must allow for adjustments (based on expert judgement) in a clear and transparent fashion. This is-from our point of view - a strong argument against the use of Neural Networks.

8. As mentioned in the last point, the effort of developing individual claims models seems only justified for sufficiently large claims portfolios. Developing a general approach-derived from available market data or possibly a large pool of actual claims portfolios - might, hence, be of particular practical interest for smaller insurance companies.

9. The starting point of our paper was the current need for actuaries to rethink many of their processes. We believe that individual claims models could be the common basis for a comprehensive approach bringing all actuarial processes together, hence reducing friction and unnecessary double work. It would be most desirable, to design the procedural landscape of an actuarial department in the 21 st century based on individual claims models, meeting the challenges actuaries are currently facing. 


\section{Conclusion}

We believe that individual claims models are the way forward for modern actuarial departments. They will yield a substantial improvement in quality (compared to standard methods on aggregated triangle data) and a better understanding of the claims portfolio and, hence, the core of the business model. However, individual claims models must not be thought of as an end in itself or as something of just actuarial interest. They must be embedded into the procedural landscape of an actuarial department and used in as many areas as possible (such as claims steering, proposals for case reserves, etc.). A successful implementation requires a clear concept and vision of all actuarial processes in their entirety and only a comprehensive approach will justify the efforts.

We have also seen that a sound understanding of the characteristics of claims as well as sufficient and detailed data are two important keys for developing individual claims models. Approaches neglecting these pivotal facts seem bound to fail. From our point of view, current discussions around individual claims models focus far too much on models or techniques and not enough on the specificities of claims. However, we believe that sound modelling requires a sound understanding of the modelling object and, hence, there should be a shift of focus in the approach to individual claims models. As indicated in the last section, this would also give rise to many interesting research questions of high practical relevance.

Funding Open Access funding enabled and organized by Projekt DEAL.

Open Access This article is licensed under a Creative Commons Attribution 4.0 International License, which permits use, sharing, adaptation, distribution and reproduction in any medium or format, as long as you give appropriate credit to the original author(s) and the source, provide a link to the Creative Commons licence, and indicate if changes were made. The images or other third party material in this article are included in the article's Creative Commons licence, unless indicated otherwise in a credit line to the material. If material is not included in the article's Creative Commons licence and your intended use is not permitted by statutory regulation or exceeds the permitted use, you will need to obtain permission directly from the copyright holder. To view a copy of this licence, visit http://creativecommons.org/licenses/by/4. $0 /$.

\section{References}

Amberger, M., John, D., Wiedemann, M.: Aufbruch in ein neues Zeitalter. Versicher. Wirtsch. 05, 68-74 (2018)

Antonio, K., Plat, R.: Micro-level stochastic loss reserving for general insurance. Scand Actuar J 07, 649-669 (2014)

Bundesgesundheitsministerium:. https://www.bundesgesundheitsministerium.de/krankenhausfinanzierung. html, Accessed 4 Dec 2021

Bundesgesundheitsministerium:. https:/www.bundesgesundheitsministerium.de/pflegegrade.html, Accessed 4 Dec 2021

Deutscher Bundestag. Drucksache 18/11397. 2017, S. 11.

Hacks, S., Wellner, W., Häcker, F., Offenloch, T.: Schmerzensgeld-Beträge 2022, 40th edn. Deutscher Anwaltverlag, (2021)

John, D., Wiedemann, M.: Actuarial Data Analytics - Der Weg zur Einzelschadenreservierung. Aktuar 04, 226-230 (2018)

Küppersbusch, G., Höher, H.O.: Ersatzansprüche bei Personenschaden, 12th edn. C. H. Beck, München (2016)

Mack, T.: Schadenversicherungsmathematik, 2nd edn. VVW, Karlsruhe (2002) 
Pardey, F.: Der Haushaltsführungsschaden, 10th edn. Verlag Versicherungswirtschaft, (2021)

Pigeon, M., Antonio, K., Denuit, M.: Individual loss reserving with the multivariate skew normal framework. ASTIN Bull. 43(3), 339-428 (2013)

Schulz-Borck, H., Hofmann, E.: Schadenersatz bei Ausfall von Hausfrauen und Müttern im Haushalt, 6th edn. Verlag Versicherungswirtschaft, (2000)

Slizyk, A.: Schmerzensgeld 2022. C. H. Beck, München (2022)

Wikipedia:. https://de.wikipedia.org/wiki/Gesetzliche_Krankenversicherung, Accessed 4 Dec 2021

Wikipedia:. https://de.wikipedia.org/wiki/Gesetzliche_Unfallversicherung_in_Deutschland, Accessed 4 Dec 2021

Wikipedia:. https://de.wikipedia.org/wiki/Pflegeversicherung_(Deutschland)., Accessed 4 Dec 2021

Wüthrich, M.: Machine learning in individual claims reserving. Scand Actuar J 06, 465-480 (2018) 